\title{
Correlated Isocurvature Fluctuation in Quintessence and Suppressed CMB Anisotropies at Low Multipoles
}

\author{
Takeo $\operatorname{Moroi}^{(a)}$ and Tomo Takahashi ${ }^{(b)}$ \\ ${ }^{(a)}$ Department of Physics, Tohoku University, Sendai 980-8578, Japan \\ (b) Department of Physics and Astronomy, University of North Carolina, Chapel Hill, NC 27599
}

\begin{abstract}
We consider cosmic microwave background (CMB) anisotropy in models with quintessence taking into account of isocurvature fluctuation in the quintessence. It is shown that, if the primordial fluctuation of the quintessence has a correlation with the adiabatic density fluctuations, CMB angular power spectrum $C_{l}$ at low multipoles can be suppressed without affecting $C_{l}$ at high multipoles. Possible scenario of generating correlated mixture of the quintessence and adiabatic fluctuations is also discussed.
\end{abstract}

Preprint number: TU-695, astro-ph/0308208

The recent measurement of the cosmic microwave background (CMB) angular power spectrum $C_{l}$ by the Wilkinson Microwave Anisotropy Probe (WMAP) [1] has greatly improved our understanding of the universe. In particular, since the CMB angular power spectrum is sensitive to the properties of the origin and evolution of the cosmic density fluctuations, now we are at the position to test various scenarios of generating cosmic density fluctuations, which requires some interplay of astrophysics, cosmology, and particle physics. In addition, the shape of the CMB angular power spectrum also depends on various cosmological parameters, and hence many of the cosmological parameters are now precisely determined.

One of the important messages from the WMAP is that our universe is well described by a low-density cold dark matter (CDM) model with (almost) flat geometry. In other words, the WMAP confirmed the existence of the dark energy which is also suggested by the observations of high red-shift Type-Ia supernovae [2].

Although the cosmological constant is the most famous candidate of the dark energy, a slowly evolving scalar field, dubbed as "quintessence" [3], is another important possibility. In models with quintessence, however, behavior of the CMB angular power spectrum may be different from that in the $\Lambda \mathrm{CDM}$ model (where the cosmological constant is assumed as the dark energy). One reason is that, since the quintessence is a dynamical scalar field, its amplitude may acquire primordial fluctuation and that isocurvature fluctuation may exist in the quintessence sector, which affects behaviors of the CMB angular power spectrum.

So far, effects of the primordial fluctuation of the quintessence have been studied only for the cases where the primordial fluctuation of the quintessence is uncorrelated with the adiabatic density fluctuations. In this case, if the quintessence-dominated universe is realized in very recent epoch as in the $\Lambda \mathrm{CDM}$ model, effects of the quintessence fluctuation on $C_{l}$ are only on very low multipoles. In addition, in the uncorrelated case, $C_{l}$ at low multipoles are always enhanced $[4,5]$. It is possible, however, that the primordial fluctuation of the quintessence has correlation with the adiabatic density fluctuations. (For explicit model of generating correlated mixture of the quintessence and adiabatic fluctuations, see the discussion below.)

Thus, in this letter, we study the CMB angular power spectrum in models with quintessence paying particular attention to the effects of the correlation of the quintessence fluctuation with adiabatic density fluctuations. If the correlation exists, behavior of the $\mathrm{CMB}$ angular power spectrum becomes different from the uncorrelated case. In particular, as we will discuss, $C_{l}$ at low multipoles may be suppressed without affecting the shape of the CMB angular power spectrum at high multipoles, which may be related to the suppression of the measured values of $C_{l}$ at low multipoles. (For other models of suppressing $C_{l}$ at low multipoles, see [6].)

Let us start with presenting the framework. Here we consider the scenario where the dark energy of the universe is given by a potential energy of a slowly evolving scalar field, quintessence $Q$; if the slow-roll condition is satisfied for the quintessence field $Q$, equation-of-state parameter of $Q$ is almost -1 and the energy density of $Q$ behaves as the cosmological constant. Although various models of quintessence have been proposed, we adopt a simple approximation of the quintessence potential; we use the parabolic potential with a constant term:

$$
V(Q)=V_{0}+\frac{1}{2} m_{Q}^{2} Q^{2} .
$$

We assume that $Q$ has non-vanishing initial amplitude. In our study, for simplicity, we consider $m_{Q}$ comparable to (or smaller than) the present expansion rate of the universe. In addition, $V_{0}$ is assumed to be of the order of the present critical density or smaller. With such a small value of $m_{Q}$ (and $V_{0}$ ), slow-roll condition for $Q$ is satisfied until very recently and energy fraction of the quintessence becomes sizable only at the very recent epoch. In this case, shape of the CMB angular power spectrum at high multipoles is almost unaffected. Notice that the above potential is a good approximation for some quintessence models, like the cosine-type one [7].

Since the quintessence is a dynamical scalar field, its amplitude may fluctuate. In particular, if its (effective) 
mass during inflation is smaller than the expansion rate, the quintessence field also acquires quantum fluctuation. Such a primordial fluctuation becomes a new source of the cosmic density fluctuations and affects the CMB angular power spectrum.

Evolution and effects of the primordial fluctuation of $Q$ have been studied for the case where the primordial fluctuation of the quintessence is not correlated with the adiabatic fluctuations $[8,4,5]$. Fluctuation of the quintessence field can be, however, correlated with the adiabatic fluctuations. If some correlation exists, effects of the primordial fluctuation of $Q$ on the CMB angular power spectrum are expected to be different from those in the uncorrelated case. Hereafter, we study the effects of quintessence fluctuation for the case where the correlation between the primordial fluctuation of $Q$ and the adiabatic fluctuations exists.

In order to parameterize the relative size of the primordial quintessence fluctuation and the adiabatic fluctuations, we define

$$
r_{Q} \equiv \frac{\delta Q_{\text {init }}}{M_{*} \Psi_{\mathrm{RD}}} .
$$

(Strictly speaking, the above expression is valid only for the case where $\delta Q_{\text {init }}$ and $\Psi_{\mathrm{RD}}$ are fully correlated. For the case where $\delta Q_{\text {init }}$ and $\Psi_{\mathrm{RD}}$ are uncorrelated, for example, it should be understood as $r_{Q}=$ $\left.\sqrt{\left\langle\delta Q_{\text {init }}^{2}\right\rangle} / M_{*} \sqrt{\left\langle\Psi_{\mathrm{RD}}^{2}\right\rangle}.\right)$ Here, $\delta Q_{\text {init }}$ is the primordial fluctuation of $Q, \Psi$ denotes the fluctuation of the $(0,0)$ component of the metric in the Newtonian gauge: $g_{00}=a^{2}(1+2 \Psi)$ with $a$ being the scale factor [9], and $M_{*}$ is the reduced Planck scale. In addition, $\Psi_{\mathrm{RD}}$ is the metric perturbation related to the adiabatic density fluctuation in the radiation-dominated epoch. We assume that $\Psi_{\mathrm{RD}}$ is (almost) scale-invariant, as suggested by the WMAP [10].

If we calculate the CMB angular power spectrum with non-vanishing values of $\delta Q_{\text {init }}$ and $\Psi_{\mathrm{RD}}$, we obtain the form

$$
C_{l}=C_{l}^{\text {(adi) }}+C_{l}^{(\text {corr })}+C_{l}^{\text {(uncorr })} .
$$

Here, $C_{l}^{(\text {adi) }}$ is the result with purely adiabatic density fluctuations. With the cosmological and model parameters used in the following analysis, $C_{l}^{(\text {adi) }}$ almost agrees with the $\mathrm{CMB}$ angular spectrum for the $\Lambda \mathrm{CDM}$ model $C_{l}^{(\Lambda \mathrm{CDM})}$. On the contrary, $C_{l}^{\text {(uncorr) }}$ is the CMB angular power spectrum purely generated from $\delta Q_{\text {init }}$, while $C_{l}^{\text {(corr) }}$ parameterizes the effects of correlation.

When $\delta Q_{\text {init }}$ and $\Psi_{\mathrm{RD}}$ are uncorrelated, $C_{l}^{(\mathrm{corr})}=0$. Furthermore, $C_{l}^{\text {(uncorr) }}$ is increased at low multipoles. As a result, the total CMB angular power spectrum may be significantly enhanced at low multipoles $[4,5]$.

If $\delta Q_{\text {init }}$ and $\Psi_{\mathrm{RD}}$ have correlation, $C_{l}^{\text {(corr) }}$ plays important roles. To see the effects of the correlation, we calculate the $\mathrm{CMB}$ angular power spectrum for the case where

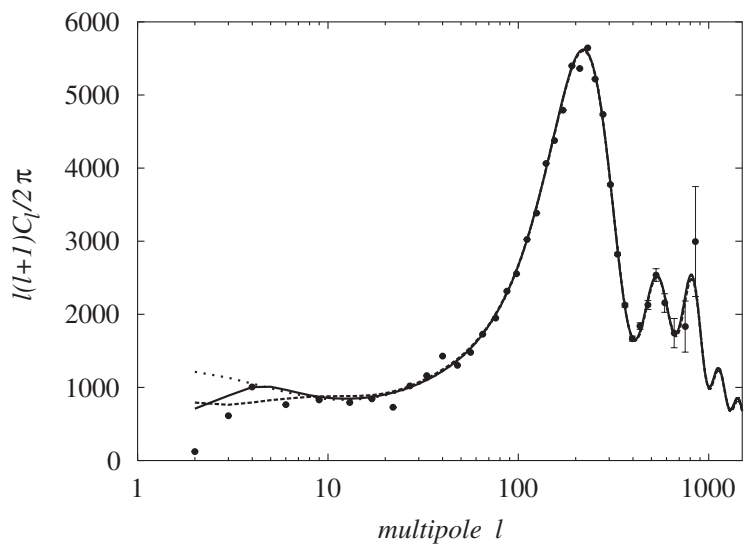

FIG. 1. The CMB angular power spectrum generated from the correlated mixture of the quintessence and adiabatic fluctuations. We take (a) $m_{Q}=10^{-42} \mathrm{GeV}, V_{0}=0$ and $r_{Q}=400$ with $\Omega_{b}=0.046, \Omega_{m}=0.27, h=0.72$, and $\tau=0.166$ [10] (solid), and (b) $m_{Q}=10^{-40} \mathrm{GeV}, V_{0}=3.0 \times 10^{-47} \mathrm{GeV}$ and $r_{Q}=2.5$ with $\Omega_{b}=0.048, \Omega_{m}=0.27, h=0.7$, and $\tau=0.2$ (dashed). (Here, $\Omega_{b}$ and $\Omega_{m}$ are density parameters of baryon and non-relativistic matter, respectively, $h$ the Hubble constant in units of $100 \mathrm{~km} / \mathrm{sec} / \mathrm{Mpc}$, and $\tau$ the reionization optical depth.) The full correlation between $\delta Q_{\text {init }}$ and $\Psi_{\mathrm{RD}}$ is assumed. Result for the purely adiabatic $\Lambda$ CDM model is also shown in the dotted line. (For cosmological parameters, we use the best-fit values suggested by the WMAP for the power-law $\Lambda \mathrm{CDM}$ model.) For comparison, we also plot the data points measured by the WMAP [12]. (The errors are measurement errors only.)

$\delta Q_{\text {init }}$ and $\Psi_{\mathrm{RD}}$ are fully correlated: $\left\langle\delta Q_{\text {init }} \Psi_{\mathrm{RD}}\right\rangle^{2}=$ $\left\langle\delta Q_{\text {init }}^{2}\right\rangle\left\langle\Psi_{\mathrm{RD}}^{2}\right\rangle$. In our numerical analysis, we always take $Q_{\text {init }}>0$. In this case, primordial fluctuation of the energy density of $Q$ has positive correlation with $\Psi_{\mathrm{RD}}$ when $r_{Q}>0$. (For details of the calculation, see $[4,5]$.)

In Fig. 1, we plot the resultant $\mathrm{CMB}$ angular power spectra for several cases with positive values of $r_{Q}$ : (a) $r_{Q}=35$ with $m_{Q}=10^{-42} \mathrm{GeV}$ and $V_{0}=0$, and (b) $r_{Q}=$ 2.5 with $m_{Q}=10^{-40} \mathrm{GeV}$ and $V_{0}=3.0 \times 10^{-47} \mathrm{GeV}$. (We checked that these data points are consistent with the recent Type 1a supernovae data [11].) Initial amplitude of the quintessence $Q_{\text {init }}$ is determined so that the present energy fraction of the quintessence becomes $\Omega_{Q}=1-\Omega_{m}=0.73$, which gives $Q_{\text {init }}=8.2 \times 10^{18} \mathrm{GeV}$ and $7.5 \times 10^{17} \mathrm{GeV}$ for the cases (a) and (b), respectively. As one can see, if $r_{Q}>0$, sizable suppression of the low multipoles is possible compared to the $\Lambda \mathrm{CDM}$ model. Notice that, in the uncorrelated case, such a suppression of $C_{l}$ at the low multipoles cannot be realized. It is also notable that, even in the correlated case, the CMB angular power spectrum at higher multipoles is almost the same as that in the $\Lambda \mathrm{CDM}$ case.

In order to see how much $C_{2}$ can be suppressed relative to the $\Lambda \mathrm{CDM}$ model, in Fig. 2, we plot the ratio 


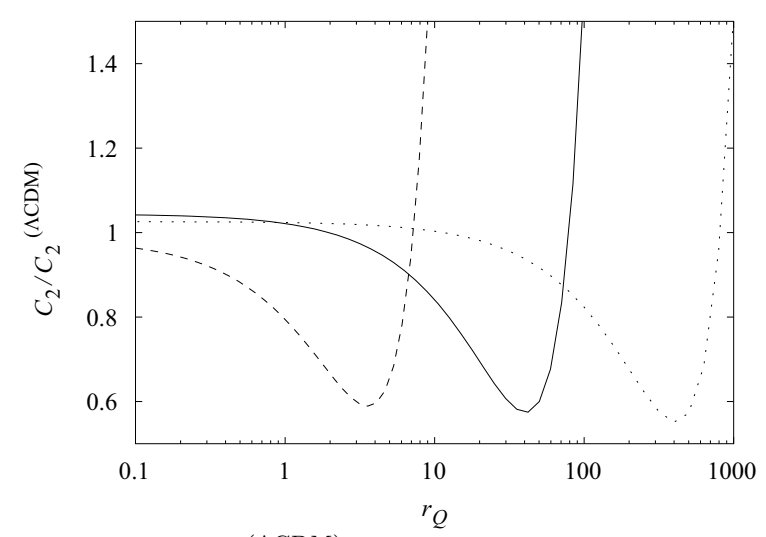

FIG. 2. $C_{2} / C_{2}^{(\Lambda \mathrm{CDM})}$ as a function of $r_{Q}$ for cases (a) and (b) given in Fig. 1 (solid and dashed, respectively), and for $m_{Q}=10^{-43} \mathrm{GeV}$ and $V_{0}=0$ (dotted) with $\Omega_{b}=0.046$, $\Omega_{m}=0.27, h=0.72$, and $\tau=0.166$. For $C_{l}^{(\Lambda \mathrm{CDM})}$, we use the best-fit values of the cosmological parameters suggested by the WMAP. The full correlation between $\delta Q_{\text {init }}$ and $\Psi_{\mathrm{RD}}$ is assumed.

$C_{2} / C_{2}^{(\Lambda \mathrm{CDM})}$ as a function of $r_{Q}$. Since $C_{l}^{(\mathrm{corr})} \propto r_{Q}$ and $C_{l}^{\text {(uncorr) }} \propto r_{Q}^{2}, C_{l}^{\text {(uncorr) }}$ is more important than $C_{l}^{\text {(corr) }}$ when $r_{Q}$ is large. In this case, the ratio $C_{2} / C_{2}^{(\Lambda \mathrm{CDM})}$ becomes larger than 1 . If $r_{Q}$ is smaller, however, $C_{l}^{\text {(corr) }}$ becomes sizable and $C_{2}$ can be suppressed. As one can see, with the correlated primordial fluctuation in the quintessence amplitude, $C_{2} / C_{2}^{(\Lambda \mathrm{CDM})}$ can be as small as $\sim 0.6$ if $r_{Q}$ is properly chosen. Importantly, effects of $\delta Q_{\text {init }}$ is limited to $C_{l}$ with small $l$ and hence the shape of the CMB angular power spectrum at higher multipoles is unchanged. Thus, the correlated fluctuation in the quintessence sector provides a new mechanism to suppress $C_{l}$ at very low multipoles without affecting the high multipoles. This may have some relevance with the suppressed values of the CMB angular power spectrum at low multipoles measured by the WMAP.

Suppression of $C_{l}$ at low multipoles improves the agreement of the theoretical prediction with the observation. To discuss this issue quantitatively, we calculate the goodness-of-fit parameter $\chi^{2}$ using the numerical program provided by the WMAP collaboration with the WMAP data [12]. Compared to the $\Lambda$ CDM model, we found that the $\chi^{2}$ variable can decrease in models with quintessence. For example, for the cases (a) and (b) given in Fig. 1, changes of the goodness-of-fit parameter are $\Delta \chi^{2}=-2.1$ and -1.2 , respectively. As can be understood from the figure, the changes in $\chi^{2}$ are only from the low multipoles.

In our study, we have also calculated $C_{l}$ with negative values of $r_{Q}$. Since $C_{l}^{(\text {corr })}$ is proportional to $r_{Q}$, the resultant $\mathrm{CMB}$ angular power spectrum is enhanced at the low multipoles when $r_{Q}<0$. Thus, if the suppression of $C_{l}$ at the low multipoles measured by the WMAP is related to the primordial fluctuation of the quintessence, positive correlation with the metric perturbation $\Psi_{\mathrm{RD}}$ (i.e., $r_{Q}>0$ ) is required in the present model.

So far, we have discussed effects of primordial fluctuations of quintessence on the CMB angular power spectrum, so we would like to comment on other cosmological perturbations in our scenario. Since the quintessence dominates the universe at very recent epoch, its effects are only on fluctuations with very large wavelength comparable to the present Hubble distance. In other words, density fluctuations with smaller wavelength are determined by the adiabatic part of the fluctuation and hence the results for those perturbations are the same as the predictions of the simple inflationary paradigm.

Finally, we present a possible scenario which generates the correlated fluctuations. We use the fact that fluctuations of two scalar fields can be correlated if they have a mixing during the inflation [13]. We consider the case with two scalar fields, $Q$ and $\phi$. (In the following, primordial fluctuation of $\phi$ becomes the dominant source of the adiabatic density fluctuations, as we will see below.) $Q$ and $\phi$ are defined as mass eigenstates in the present universe. In the early universe (i.e., for example, during inflation), however, Hubble-induced interaction may cause a mixing between $Q$ and $\phi$ and hence the mass eigenstates may be linear combinations of them. We denote the mass eigenstates as $\xi$ and $\eta$, and define the mixing angle $\theta$ as

$$
\left(\begin{array}{l}
\xi \\
\eta
\end{array}\right)=\left(\begin{array}{cc}
\cos \theta(t) & -\sin \theta(t) \\
\sin \theta(t) & \cos \theta(t)
\end{array}\right)\left(\begin{array}{l}
Q \\
\phi
\end{array}\right) .
$$

In our model, $\theta(t)$ varies from non-vanishing value during inflation $\theta_{\text {inf }}$ to the present value $\theta_{\text {now }}=0$. If the (effective) mass of $\eta$ is large during inflation while that of $\xi$ is negligible, then only the $\xi$ field acquires the quantum fluctuation:

$$
\delta \xi_{\mathrm{inf}}=\frac{H_{\mathrm{inf}}}{2 \pi}, \quad \delta \eta_{\mathrm{inf}}=0,
$$

where $H_{\mathrm{inf}}$ is the expansion rate during the inflation. Assuming that $\theta(t)$ rapidly changes from $\theta_{\text {inf }}$ to 0 at the end of inflation, primordial fluctuation of $Q$ and $\phi$ are given by

$$
\delta Q_{\text {init }}=\delta \xi_{\text {inf }} \cos \theta_{\text {inf }}, \quad \delta \phi_{\text {init }}=\delta \xi_{\text {inf }} \sin \theta_{\text {inf }},
$$

and correlated fluctuations are generated in the quintessence and $\phi$ fields.

The above situation may be realized if the potential of the scalar fields is of the form

$$
V=V_{0}+\frac{1}{2} m_{Q}^{2} Q^{2}+\frac{1}{2} m_{\phi}^{2} \phi^{2}+V_{\text {Hubble }}
$$

where

$$
V_{\text {Hubble }}=H_{\mathrm{vac}}^{2}\left(Q \sin \theta_{\mathrm{inf}}+\phi \cos \theta_{\mathrm{inf}}\right)^{2} .
$$


Here, $V_{\text {Hubble }}$ is the Hubble-induced interaction which is effective only during the inflation with $H_{\mathrm{vac}}$ being the expansion rate of the universe induced by the "vacuum energy;" $H_{\mathrm{vac}}=H_{\mathrm{inf}}$ and $H_{\mathrm{vac}} \simeq 0$ for during and after the inflation, respectively. With this potential, one of the mass eigenstates $\eta \simeq \phi+\theta_{\text {inf }} Q$ acquires an effective mass comparable to the expansion rate and its quantum fluctuation during inflation becomes negligibly small. Other mass eigenstate $\xi \simeq Q-\theta_{\text {inf }} \phi$, on the contrary, stays almost massless and it acquires the quantum fluctuation.

If the decay rate of the inflaton field is larger than $m_{\phi}$, slow roll condition is satisfied for $\phi$ at the time of the inflaton decay. In this case, $\delta \phi_{\text {init }}$ may become the dominant source of the adiabatic density fluctuations. In order to generate the adiabatic fluctuations from the fluctuation of $\phi$, we can use the curvaton mechanism [14] where the primordial fluctuation of the curvaton, a latedecaying scalar field, becomes the dominant source of the adiabatic density fluctuations. (Here, we do not identify $\phi$ as inflaton; in our model, $\phi$ should acquire large effective mass during inflation and hence $\phi$ cannot be the inflaton.) Indeed, if the energy density of $\phi$ once dominates the universe, $\phi$ plays the role of curvaton and the metric perturbation in the radiation dominated epoch is given by [15]

$$
\Psi_{\mathrm{RD}}=-\frac{4}{9} \frac{\delta \phi_{\text {init }}}{\phi_{\text {init }}}
$$

where $\phi_{\text {init }}$ is the initial amplitude of $\phi$ determined during the inflation. As a result, with Eq. (6), correlated mixture of adiabatic and quintessence fluctuations is generated. In this model, the $r_{Q}$ parameter is estimated as

$$
r_{Q}=\frac{1}{2 \pi} \frac{H_{\mathrm{inf}}}{M_{*} \Psi_{\mathrm{RD}}} \cos \theta_{\mathrm{inf}} .
$$

Notice that, using $\Psi_{\mathrm{RD}} \sim O\left(10^{-5}\right)$ and the upper bound $H_{\text {inf }} / M_{*} \lesssim 7 \times 10^{-5}[16], r_{Q} \lesssim 1$ in this simple model. Larger value of $r_{Q}$ is, however, possible if we extend the model. For example, if the coefficient of the kinetic term of $Q$ varies after the inflation, value of $\delta Q$ (for the canonically normalized field) also changes. Including this effect,

$$
r_{Q}=Z_{Q, \mathrm{inf}}^{-1 / 2} \frac{H_{\mathrm{inf}} / 2 \pi}{M_{*} \Psi_{\mathrm{RD}}} \cos \theta_{\mathrm{inf}},
$$

where $Z_{Q \text {,inf }}$ is the coefficient of the kinetic term of $Q$ during the inflation. (We normalize the present value of $Z_{Q}$ to be 1.) Thus, if $Z_{Q \text {,inf }}<1, r_{Q}$ can be enhanced.

In summary, we have seen that the CMB angular power spectrum at small $l$ can be suppressed without affecting that at higher multipoles in models with quintessence, if the primordial fluctuation of the quintessence has correlation with the adiabatic density fluctuations. We have also pointed out that such a correlation may be generated during inflation if the quintessence field has some mixing with other scalar field which is responsible for generating the adiabatic density fluctuations.
Acknowledgments: We acknowledge the use of CMBFAST [17] package for our numerical calculations. T.T. thanks High Energy Theory Group in Tohoku University, where this work has been done, for their hospitality during the visit. The work of T.M. is supported by the Grant-in-Aid for Scientific Research from the Ministry of Education, Science, Sports, and Culture of Japan, No. 15540247. The work of T.T. is supported by the US Department of Energy under Grant No. DE-FG02-97ER41036 .

[1] C. Bennett et al., arXiv:astro-ph/0302208.

[2] A. G. Riess et al., Astron. J. 116 (1998) 1009; S. Perlmutter et al., Astrophys. J. 517 (1999) 565.

[3] P. J. Peebles and B. Ratra, Astrophys. J. 325 (1988) L17; B. Ratra and P. J. Peebles, Phys. Rev. D 37 (1988) 3406; R. R. Caldwell, R. Dave and P. J. Steinhardt, Phys. Rev. Lett. 80 (1998) 1582; S. M. Carroll, Phys. Rev. Lett. 81 (1998) 3067; I. Zlatev, L. M. Wang and P. J. Steinhardt, Phys. Rev. Lett. 82 (1999) 896.

[4] M. Kawasaki, T. Moroi and T. Takahashi, Phys. Rev. D 64 (2001) 083009.

[5] M. Kawasaki, T. Moroi and T. Takahashi, Phys. Lett. B 533 (2002) 294.

[6] C. R. Contaldi, M. Peloso, L. Kofman and A. Linde, JCAP 0307 (2003) 002; J. M. Cline, P. Crotty and J. Lesgourgues, arXiv:astro-ph/0304558; B. Feng and X. Zhang, arXiv:astro-ph/0305020; M. Kawasaki and F. Takahashi, arXiv:hep-ph/0305319; M. BasteroGil, K. Freese and L. Mersini-Houghton, arXiv:hepph/0306289; N. Kaloper and M. Kaplinghat, arXiv:hepth/0307016; S. Tsujikawa, R. Maartens and R. Brandenberger, arXiv:astro-ph/0308169.

[7] J. E. Kim, JHEP 9905 (1999) 022.

[8] L. R. Abramo and F. Finelli, Phys. Rev. D 64 (2001) 083513.

[9] H. Kodama and M. Sasaki, Prog. Theor. Phys. Suppl. 78 (1984) 1.

[10] D. N. Spergel et al., arXiv:astro-ph/0302209.

[11] J. L. Tonry et al., Astrophys. J. 594 (2003) 1

[12] G. Hinshaw et al., arXiv:astro-ph/0302217.

[13] C. Gordon, D. Wands, B. A. Bassett and R. Maartens, Phys. Rev. D 63 (2001) 023506.

[14] S. Mollerach, Phys. Rev. D 42 (1990) 313; A. D. Linde and V. Mukhanov, Phys. Rev. D 56 (1997) 535; K. Enqvist and M. S. Sloth, Nucl. Phys. B 626 (2002) 395; D. H. Lyth and D. Wands, Phys. Lett. B 524 (2002) 5; T. Moroi and T. Takahashi, Phys. Lett. B 522 (2001) 215 [Erratum-ibid. B 539 (2002) 303].

[15] T. Moroi and T. Takahashi, in Ref. [14]; Phys. Rev. D 66 (2002) 063501.

[16] H. V. Peiris et al., Astrophys. J. Suppl. 148 (2003) 213; V. Barger, H. S. Lee and D. Marfatia, Phys. Lett. B 565 (2003) 33; S. M. Leach and A. R. Liddle, arXiv:astro$\mathrm{ph} / 0306305$.

[17] U. Seljak and M. Zaldarriaga, Astrophys. J. 469 (1996) 437. 